\title{
Usefulness of Oblique Lumbar Magnetic Resonance Imaging for Nerve Root Anomalies and Extraforaminal Entrapment Lesions
}

\author{
Dong Hwa Heo ${ }^{1}$, Seok Woo Kim ${ }^{2}$, Hsien-Hao Chang ${ }^{1}$, Choon-Keun Park ${ }^{1}$ \\ ${ }^{1}$ Department of Neurosurgery, Spine Center, Spine Research Institute, The Leon Wiltse Memorial Hospital, Suwon, Korea \\ ${ }^{2}$ Department of Orthopedics, Spine Center, Hallym University Sacred Heart Hospital, Anyang, Korea
}

Study Design: A retrospective review of prospectively evaluated magnetic resonance (MR) images.

Purpose: Routine lumbar axial and sagittal MR images may not clearly demonstrate nerve root anomalies and entrapments in the extraforaminal region. Thus, lumbar extraforaminal lesions or nerve root anomalies may be underdiagnosed because of unfamiliar radiological anatomy. We aimed to investigate the clinical efficacy of our oblique magnetic resonance imaging (MRI) technique for diagnosing nerve root anomalies and entrapment lesions.

Overview of Literature: Evaluation of clinical usefulness of oblique lumbar MRI for nerve root anomalies and extraforaminal entrapment lesions.

Methods: In total, 162 patients ( 60 males and 102 females; mean age, 59.8 17.8 years) with suspected nerve root anomalies and entrapments in routine axial and sagittal MR images underwent unilateral or bilateral oblique lumbar T2-weighted MRI. The axial angle of the oblique image was parallel to the foramen. The oblique MRI findings of the symptomatic side were compared with those of the asymptomatic side in cases with unilateral pathologic lesions. Interobserver agreement was analyzed using kappa statistics.

Results: The following abnormal findings were obtained: nerve root entrapment due to foraminal stenosis in 82 cases; extraforaminal disk herniation in 29; conjoined nerve root in six; and foraminal bony cyst in one. Forty-three of the 46 patients experienced unilateral lateralizing symptoms, unilateral nerve root entrapment due to foraminal stenosis, herniated disk, and extraforaminal bony cyst compared with the asymptomatic contralateral side $(p<0.05)$.

Conclusions: Our results suggest that oblique lumbar MRI is a simple and valuable modality for diagnosing anomalous lumbar nerve root lesions and entrapment.

Keywords: Radiculopathy; Anomaly; Lumbar; Magnetic resonance imaging

\section{Introduction}

Routine lumbar axial and sagittal magnetic resonance imaging (MRI) have failed to clearly demonstrate nerve root anomalies and entrapment in the extraforaminal area [1-
3], which deterred the diagnosis of conditions such as extraforaminal lumbar disk herniation and foraminal stenosis $[3,4]$. Nerve root anomalies are of clinical importance for predicting the risk of injury during spinal surgery $[5,6]$, particularly during minimally invasive spinal surgeries

Received Aug 19, 2017; Revised Sep 16, 2017; Accepted Oct 7, 2017

Corresponding author: Seok Woo Kim

Department of Orthopedics, Spine Center, Hallym University Sacred Heart Hospital, 22 Gwanpyeong-ro 170beon-gil, Dongan-gu, Anyang 14068, Korea

Tel: +82-82-31-380-6000, Fax: +82-31-380-4118, E-mail: seokwookim@yahoo.com 
such as percutaneous endoscopic procedures and transforaminal lumbar interbody fusion. Furthermore, diagnosis of nerve root entrapment is crucial for optimal treatment [7].

We suggest that oblique lumbar MRI can clearly demonstrate the pedicle, nerve root, ganglion, and radiological anatomy of the foraminal and extraforaminal regions. Here, we present a technical oblique lumbar MRI method and assess its efficacy in diagnosing nerve root anomalies and entrapment lesions.

\section{Materials and Methods}

Since 2013, a total of 162 patients underwent initial routine axial and sagittal MRI at the Leon Wiltse Memorial Hospital. Upon suspicion of lumbar nerve root entrapment or anomalous lesions, oblique unilateral or bilateral lumbar MRI was performed. We retrospectively analyzed the oblique MRI data. This study was approved by the Institutional Review Board of the Leon Wiltse Memorial Hospital (IRB approval no., \#2015-05) and conforms to the tenets of the Declaration of Helsinki.

\section{Indications of oblique lumbar magnetic resonance imaging}

Oblique lumbar MRI was additionally performed in patients suspected of having the following diseases or diagnoses upon routine axial and sagittal MRI: (1) foraminal stenosis, (2) nerve root anomalies (furcal and conjoined nerve roots), (3) extraforaminal disc herniations, and (4) nerve root entrapment by various pathologic lesions of foraminal and extraforaminal regions.

\section{Procedure of oblique lumbar magnetic resonance imaging}

Oblique lumbar MRI was performed with a 1.5-T MRI system (Signa Excite; GE Healthcare, Milwaukee, WI, USA) using a T2-weighted sequence (repetition time, 2,750 ms; echo time, $113 \mathrm{~ms}$; slice thickness, $2 \mathrm{~mm}$; flip angle, 90 ; field of view, $240 \times 240$ ).

Oblique lumbar MRI was modified for the coronal images. The sagittal line of the oblique image was parallel to the line connecting the posterior margin of the upper end plate to the lower end plate of the affected level's functional segmental unit (Fig. 1A) [4]. For example, in the case of an L4-5 lesion, the sagittal line was a line connecting the posterior margin of the upper end plate of $\mathrm{L} 4$ to the lower end plate of L5. The axial angle of the oblique image was parallel to the foramen (Fig. 1B) [4]. In our experience, the axial angle optimally ranges from $17^{\circ}$ to $23^{\circ}$.

\section{Analysis of oblique magnetic resonance images}

Two different clinicians assessed the oblique lumbar MR images. In cases of unilateral pathologic lesions, oblique MRI findings of the symptomatic side were compared


Fig. 1. Magnetic resonance (MR) images of a 52-year-old female with severe pain in the right leg. Trajectory and angle of oblique lumbar magnetic resonance imaging. (A) Sagittal line of oblique image (black line) is parallel to the line from the posterior margin of the upper to lower end plate of the functional segmental unit in the affected level. (B) Axial angle of oblique image (black line) is parallel to the foramen. Axial T1-weighted MR image indicates ruptured disc particles in the right extraforaminal region of $L 4-5$ (arrow). (C) Lumbar oblique MR image indicate each lumbar nerve root obliquely passing under the pedicles. Right side T2-weighted oblique MR image indicates the cut off of the exiting $L 4$ nerve root by the ruptured disc at the extraforaminal area (arrow). 
with those of the asymptomatic side. If the patients presented with unilateral lateralizing symptoms or signs, such as unilateral radiating pain or weakness of the legs, oblique MRI findings of the symptomatic side were compared with those of the asymptomatic side. The exiting nerve root entrapment caused by foraminal stenosis or extraforaminal herniated disc particles in the symptomatic side was compared with that of the asymptomatic contralateral side. Furthermore, the abnormal findings below and above the nerve roots were compared.

Data are presented as mean \pm standard deviation. Fisher's exact test and interobserver agreement (weighted kappa value) were used for statistical analysis using the $\mathrm{R}$ software (https://www.r-project.org/).

\section{Results}

In total, 162 patients (60 males and 102 females; mean age, $59.8 \pm 17.8$ years) were enrolled in our study (see
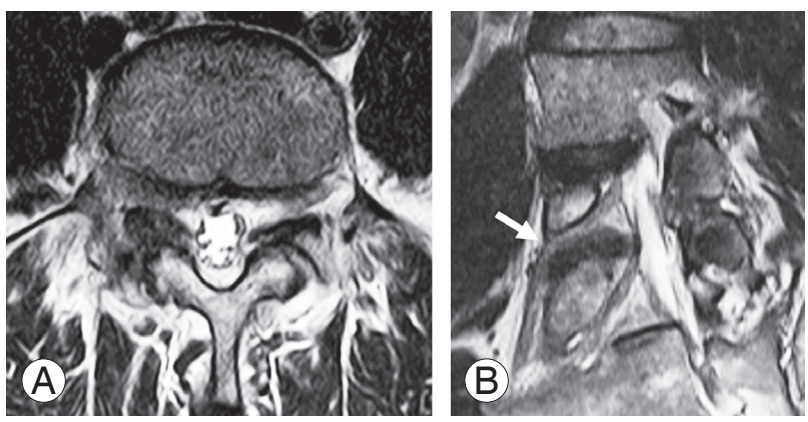

Fig. 2. A 38-year-old male presented with pain in the back and right leg. T2-weighted magnetic resonance images indicate isthmic spondylolisthesis and right foraminal stenosis with pseudo-disc bulging of L5-S1 (A) and entrapment of the right $L 5$ nerve root (B, arrow).
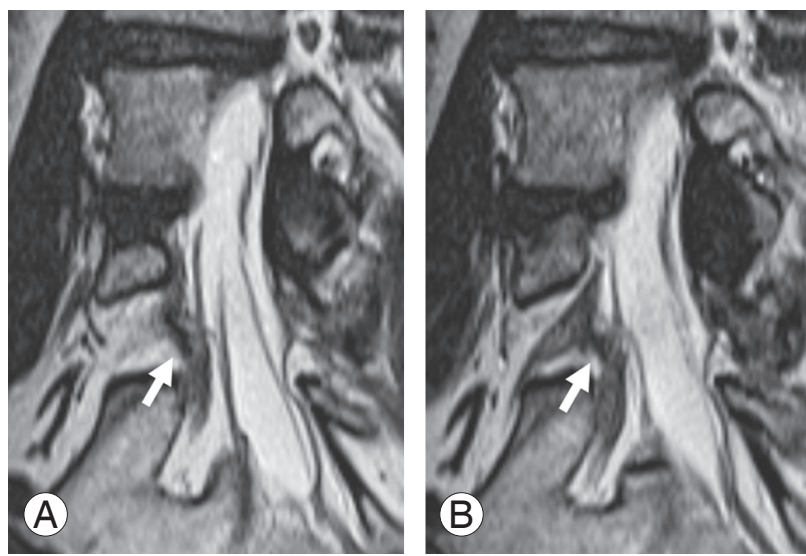

Fig. 3. Oblique magnetic resonance (MR) image of a 52-year-old female with back pain. (A, B) Serial oblique MR images indicate right side L5 and S1 conjoined nerve root (arrows).
Table 1 for patient characteristics). Of these, 118 (72.8\%) displayed the following abnormal findings: extraforaminal disc herniation in 29 cases (Fig. 1), nerve root entrapment due to foraminal stenosis in 82 (Fig. 2), conjoined nerve root in six (L5 and S1 in four cases, S1 and S2 in two cases) (Table 1, Fig. 3), and foraminal bony cyst in 1 (Fig. 4).

Oblique lumbar MRI clearly demonstrated the pedicles, dorsal root ganglia, and lumbar nerve roots of the foraminal and extraforaminal regions; however, clear images could not be obtained for four patients with degenerative scoliosis and spondylosis. Forty-six patients presented with lateralizing unilateral symptoms such as unilateral radicular pain or weakness. In 43 of the 46 patients (93.5\%), unilateral nerve root entrapment due to unilateral foraminal stenosis, herniated disc, or extraforaminal bony cyst were clearly observed when compared with the asymptomatic contralateral side $(p<0.05)$. Interobserver agreement was observed to be excellent for oblique MRI

Table 1. Patient characteristics ( $N=162$ )

\begin{tabular}{lc} 
Characteristic & Value \\
\hline Age (yr) & $59.8 \pm 17.8$ \\
Male/female & $60 / 102$ \\
\hline Diagnosis & $118(72.8)$ \\
\hline Foraminal stenosis & 82 \\
\hline Extraforaminal disc hemiation & 29 \\
Conjoined nerve root & 6 \\
\hline Foraminal bony cyst & 1 \\
\hline
\end{tabular}

Values are presented as mean \pm standard deviation or number (\%).
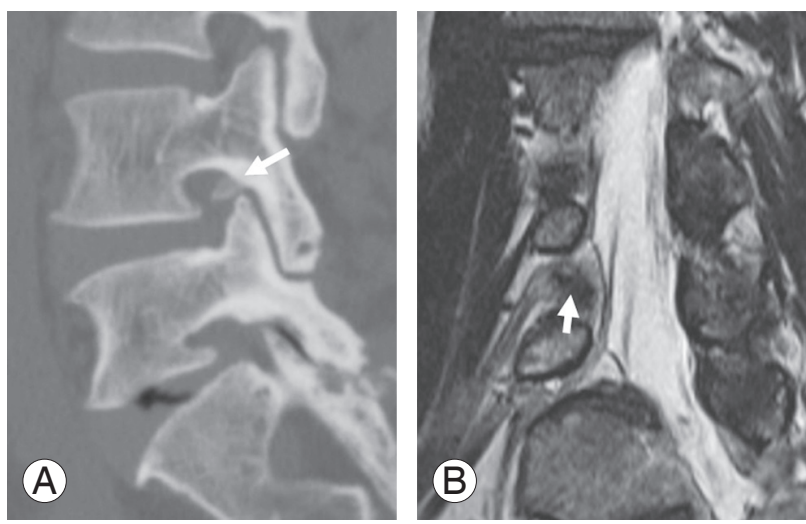

Fig. 4. Radiologic image of a 56-year-old male with right radiculopathy. (A) Sagittal reconstruction computed tomographic image indicates a bony cyst at the right intervertebral foramen of $L 4-5$ (arrow). (B) Right side T2-weighted oblique magnetic resonance imaging indicates compression of $\mathrm{L} 4$ nerve root by bony cyst (arrow). 
Table 2. Interobserver reliability of oblique lumbar magnetic resonance imaging (kappa values)

\begin{tabular}{lccc} 
Observers & First reading & Second reading & Overall \\
Reader 1 and 2 & 0.84 & 0.92 & 0.88 \\
\hline
\end{tabular}

(overall kappa value $=0.88)$ (Table 2$)$.

\section{Discussion}

A centrally herniated lumbar disc of the spinal canal can be easily diagnosed using routine axial and sagittal MRI or computed tomography. However, it may be challenging to precisely detect foraminal and extraforaminal lesions, and thus, lead to overlooks on routine axial and sagittal lumbar MRI $[3,4,8]$. Recently, attempts have been made to use three-dimensional (3D) MRI and 3D MR rendering imaging to evaluate lumbosacral foraminal and extraforaminal lesions $[2,3,8,9]$. Coronal 3D MRI images have accurately demonstrated the foraminal and extraforaminal regions of the lumbosacral nerve roots and have precisely diagnosed foraminal stenosis and extraforaminal disc herniation [2,3]. Furthermore, diffusion-tensor imaging can help visualize nerve fiber tracts, foraminal stenosis, and extraforaminal disc herniation [8].

The exiting lumbosacral spinal nerve root passes obliquely through the intervertebral foramen in an inferior-ventral direction [10-12]. Therefore, axial, sagittal, and coronal MRI may not precisely demonstrate the spinal nerve root in the extraforaminal region. Conventional foraminal MRI is taken at a right angle to the foramen; thus, foraminal MRI comprises oblique sagittal images. In contrast, our oblique MRI was taken parallel to the foramen and close to the oblique coronal plane. Oblique lumbar MRI was based on the radiological anatomy of the spinal nerve roots. Our findings suggest that oblique MR images can demonstrate foraminal and extraforaminal regions.

In T2-weighted images, the degenerative disc signal was lower than the nerve root signal. Therefore, we performed oblique T2-weighted MRI to detect extraforaminal nerve root compression caused by extraforaminal disc herniation [4]. Because oblique MRI helped compare the abnormal findings between the regions above and below the nerve roots on the symptomatic side as well as the contralateral asymptomatic side, root entrapment or anomalous lesions could be easily detected. Oblique MRI data were easily interpreted with good interobserver agreement.
Oblique MRI did not require any additional programs or intravenous injections and could be performed using the 1.5-T MRI scanner.

Despite the advantages of oblique lumbar MRI, the foraminal and extraforaminal radiological anatomy of patients with spinal deformities cannot be demonstrated clearly. Furthermore, it is difficult to determine the axial and sagittal angles of oblique MRI; these angles should be changed in accordance with level due to spinal deformity [4]. Therefore, oblique MRI may not provide adequate information regarding the foraminal and extraforaminal region in cases with scoliosis or hyper-lordosis. Thus, coronal 3D MRI may be more suitable than the presently used oblique MRI for patients with spinal deformities.

Axial and sagittal MRI may not precisely demonstrate anomalous root lesions; hence, spinal nerve root anomalies may be underdiagnosed $[5,6]$. Although the incidence of nerve root anomalies is very low, anomalous nerve root lesions can interfere with spinal operations. Incomplete decompression or root injury can occur during lumbar spine surgery in cases with unsuspected root anomalies. Here, conjoined nerve roots were clearly detected using oblique lumbar MRI (Fig. 3). Moreover, we detected furcal nerve roots using this technique in a previous study [4]. We suggest that additional oblique MRI can help diagnose nerve root anomalies and provide guidance with respect to their surgical treatment.

\section{Conclusions}

Oblique lumbar MRI is a probable simple and useful diagnostic tool for root anomalies, such as conjoined or furcal nerve root, and spinal nerve entrapments, such as extraforaminal lumbar disc herniation and foraminal stenosis.

\section{Conflict of Interest}

No potential conflict of interest relevant to this article was reported.

\section{References}

1. Montinaro A. The microsurgical approach to extraforaminal lumbar disc herniations: an analysis of 15 cases. J Neurosurg Sci 2004;48:23-8.

2. Yamada $\mathrm{H}$, Terada $\mathrm{M}$, Iwasaki $\mathrm{H}$, et al. Improved accuracy of diagnosis of lumbar intra and/or extra- 
foraminal stenosis by use of three-dimensional MR imaging: comparison with conventional MR imaging. J Orthop Sci 2015;20:287-94.

3. Byun WM, Jang HW, Kim SW. Three-dimensional magnetic resonance rendering imaging of lumbosacral radiculography in the diagnosis of symptomatic extraforaminal disc herniation with or without foraminal extension. Spine (Phila Pa 1976) 2012;37:840-4.

4. Heo DH, Lee MS, Sheen SH, Cho SM, Cho YJ, Oh SM. Simple oblique lumbar magnetic resonance imaging technique and its diagnostic value for extraforaminal disc herniation. Spine (Phila Pa 1976) 2009;34:2419-23.

5. Burke SM, Safain MG, Kryzanski J, Riesenburger RI. Nerve root anomalies: implications for transforaminal lumbar interbody fusion surgery and a review of the Neidre and Macnab classification system. Neurosurg Focus 2013;35:E9.

6. Trimba R, Spivak JM, Bendo JA. Conjoined nerve roots of the lumbar spine. Spine J 2012;12:515-24.

7. Morishita Y, Ohta H, Matsumoto Y, Shiba K, Naito
M. Intra-operative identification of conjoined lumbosacral nerve roots: a report of three cases. J Orthop Surg (Hong Kong) 2012;20:90-3.

8. Kitamura M, Eguchi Y, Inoue G, et al. A case of symptomatic extra-foraminal lumbosacral stenosis ("far-out syndrome") diagnosed by diffusion tensor imaging. Spine (Phila Pa 1976) 2012;37:E854-7.

9. Filler AG, Haynes J, Jordan SE, et al. Sciatica of nondisc origin and piriformis syndrome: diagnosis by magnetic resonance neurography and interventional magnetic resonance imaging with outcome study of resulting treatment. J Neurosurg Spine 2005;2:99115 .

10. Jenis LG, An HS. Spine update: lumbar foraminal stenosis. Spine (Phila Pa 1976) 2000;25:389-94.

11. Guvencer M, Naderi S, Kiray A, Yilmaz HS, Tetik S. The relation between the lumbar vertebrae and the spinal nerves for far lateral lumbar spinal approaches. J Clin Neurosci 2008;15:192-7.

12. Bae HG, Choi SK, Joo KS, et al. Morphometric aspects of extraforaminal lumbar nerve roots. Neurosurgery 1999;44:841-6. 